\title{
Evaluation of dosimetric effect caused by slowing with multi-leaf collimator (MLC) leaves for volumetric modulated arc therapy (VMAT)
}

\author{
Zhengzheng $\mathrm{Xu}^{1,2}$, Iris Z. Wang ${ }^{1,2}$, Lalith K. Kumaraswamy ${ }^{1}$, Matthew B. Podgorsak ${ }^{1,2}$ \\ ${ }^{1}$ Department of Radiation Medicine, Roswell Park Cancer Institute, Buffalo, NY 14263 \\ ${ }^{2}$ Department of Physiology and Biophysics, State University of New York at Buffalo, Buffalo, NY 14214
}

Radiol Oncol 2016; 50(1): 121-128.

Received: 16 June 2015

Accepted: 19 October 2015

Correspondence to: Zhengzheng Xu, Department of Radiation Medicine, Roswell Park Cancer Institute, Elm and Carlton Streets, Buffalo, NY, USA, 14263. Phone: + 1919724 7764; Email: zhengzheng.xu@roswellpark.org

Disclosure: The authors declare no conflict of interest.

Background. This study is to report 1) the sensitivity of intensity modulated radiation therapy (IMRT) QA method for clinical volumetric modulated arc therapy (VMAT) plans with multi-leaf collimator (MLC) leaf errors that will not trigger MLC interlock during beam delivery; 2) the effect of non-beam-hold MLC leaf errors on the quality of VMAT plan dose delivery.

Materials and methods. Eleven VMAT plans were selected and modified using an in-house developed software. For each control point of a VMAT arc, MLC leaves with the highest speed $(1.87-1.95 \mathrm{~cm} / \mathrm{s})$ were set to move at the maximal allowable speed $(2.3 \mathrm{~cm} / \mathrm{s})$, which resulted in a leaf position difference of less than $2 \mathrm{~mm}$. The modified plans were considered as 'standard' plans, and the original plans were treated as the 'slowing MLC' plans for simulating 'standard' plans with leaves moving at relatively lower speed. The measurement of each 'slowing MLC' plan using MapCHECK ${ }^{\circledR}$ was compared with calculated planar dose of the 'standard' plan with respect to absolute dose Van Dyk distance-to-agreement (DTA) comparisons using $3 \% / 3 \mathrm{~mm}$ and $2 \% / 2 \mathrm{~mm}$ criteria.

Results. All 'slowing MLC' plans passed the $90 \%$ pass rate threshold using $3 \% / 3 \mathrm{~mm}$ criteria while one brain and three anal VMAT cases were below $90 \%$ with $2 \% / 2 \mathrm{~mm}$ criteria. For ten out of eleven cases, DVH comparisons between 'standard' and 'slowing MLC' plans demonstrated minimal dosimetric changes in targets and organs-at-risk.

Conclusions. For highly modulated VMAT plans, pass rate threshold $(90 \%)$ using $3 \% / 3 \mathrm{~mm}$ criteria is not sensitive in detecting MLC leaf errors that will not trigger the MLC leaf interlock. However, the consequential effects of non-beam hold MLC errors on target and OAR doses are negligible, which supports the reliability of current patient-specific IMRT quality assurance (QA) method for VMAT plans.

Key words: VMAT; MLC; MapCHECK2; quality assurance; DVH

\section{Introduction}

Volumetric modulated arc therapy (VMAT) demands high level of precision and reliability from the linear accelerator (LINAC) control system because the gantry rotation is synchronized with multi-leaf collimator (MLC) movement for accurate dose delivery. ${ }^{1,2}$ Highly modulated dose distribution commonly requires MLC leaves of high speed moving along the whole arc. ${ }^{3-5}$ However, fast leaf motion during gantry rotation may be affected by interleaf friction or MLC motor problems that result in leaf position errors. ${ }^{6}$ Wijesooriya et al and Ling et al reported an increase in MLC leaf position errors due to fast moving leaves. 3,7

Detecting dosimetric variation caused by MLC leaf errors is one important concern in patientspecific quality assurance (QA). Currently, several dose measuring systems are available for patientspecific QA. ${ }^{8-11}$ Fredh et al. evaluated the dosimetric effect of MLC position error on four single arc plans using Delta $4^{\circledR}$, OCTAVIUS $^{\circledR}$, COMPASS $^{\circledR}$ and 
TABLE 1. Volumetric modulated arc therapy (VMAT) plan parameters (dose, gantry speed, Leaf travel and modulation complexity score [LTMCS] and arcs), ranges of the maximal leaf speed, multi-leaf collimator (MLC) leaf position changes along 178 control points (CPs), and total modified leaves percentage of each VMAT arc

\begin{tabular}{|c|c|c|c|c|c|c|c|}
\hline Case $^{a}$ & $\begin{array}{c}\text { Dose } \\
\text { Prescription } \\
(G y / f x \times f x) \\
\end{array}$ & $\begin{array}{l}\text { Gantry } \\
\text { speed } \\
\text { (deg/s) }\end{array}$ & LTMCS $^{b}$ & Arc & $\begin{array}{l}\text { Maximal MLC leaf } \\
\text { speed range along } \\
178 \mathrm{CPs}(\mathrm{cm} / \mathrm{s})\end{array}$ & $\begin{array}{c}\text { Range of Leaf } \\
\text { Position Changes } \\
\text { along } 178 \mathrm{CPs}(\mathrm{mm})\end{array}$ & $\begin{array}{c}\text { Total Modified MLC } \\
\text { leaves \% }\end{array}$ \\
\hline Pl & $2.0 \times 33$ & 4.8 & 0.164 & 1 & $1.87-1.92$ & $-1.90-1.88$ & 1.5 \\
\hline \multirow[t]{2}{*}{ P2 } & $2.0 \times 33$ & 4.8 & 0.262 & 1 & $1.87-1.90$ & $-1.90-2.00$ & 1.6 \\
\hline & & & & 2 & $1.87-1.90$ & $-1.90-2.00$ & 1.6 \\
\hline \multirow[t]{2}{*}{ P3 } & $2.0 \times 33$ & 4.8 & 0.163 & 1 & $1.87-1.90$ & $-1.90-2.00$ & 1.5 \\
\hline & & & & 2 & $1.87-1.90$ & $-1.90-2.00$ & 1.6 \\
\hline BI & $1.8 \times 33$ & 4.8 & 0.204 & 1 & $1.87-1.92$ & $-1.88-1.90$ & 1.6 \\
\hline \multirow[t]{2}{*}{ B2 } & $1.8 \times 33$ & 4.8 & 0.199 & 1 & $1.87-1.92$ & $-1.88-1.90$ & 1.5 \\
\hline & & & & 2 & $1.87-1.92$ & $-1.88-1.90$ & 1.7 \\
\hline \multirow[t]{2}{*}{ B3 } & $1.8 \times 33$ & 4.8 & 0.217 & 1 & $1.85-1.90$ & $-1.88-2.00$ & 1.5 \\
\hline & & & & 2 & $1.85-1.90$ & $-1.88-2.00$ & 1.6 \\
\hline \multirow[t]{4}{*}{$\mathrm{Al}$} & $1.8 \times 33$ & 4.8 & 0.081 & 1 & $1.90-1.92$ & $-1.80-1.80$ & 1.7 \\
\hline & & & & 2 & $1.90-1.92$ & $-1.80-1.80$ & 1.7 \\
\hline & & & & 3 & $1.90-1.92$ & $-1.80-1.80$ & 1.7 \\
\hline & & & & 4 & $1.90-1.92$ & $-1.80-1.80$ & 1.8 \\
\hline \multirow[t]{4}{*}{ A2 } & $1.8 \times 33$ & 4.8 & 0.083 & 1 & $1.92-1.95$ & $-1.87-1.87$ & 1.5 \\
\hline & & & & 2 & $1.92-1.95$ & $-1.87-1.87$ & 1.6 \\
\hline & & & & 3 & $1.92-1.95$ & $-1.87-1.87$ & 1.6 \\
\hline & & & & 4 & $1.92-1.95$ & $-1.87-1.87$ & 1.5 \\
\hline \multirow[t]{3}{*}{ A3 } & $1.8 \times 33$ & 4.8 & 0.105 & 1 & $1.92-1.95$ & $-1.87-1.87$ & 1.7 \\
\hline & & & & 2 & $1.92-1.95$ & $-1.87-1.87$ & 1.7 \\
\hline & & & & 3 & $1.92-1.95$ & $-1.87-1.87$ & 1.7 \\
\hline \multirow[t]{4}{*}{ A4 } & $1.8 \times 33$ & 4.8 & 0.076 & 1 & $1.87-1.90$ & $-1.80-1.93$ & 2.2 \\
\hline & & & & 2 & $1.87-1.90$ & $-1.80-1.93$ & 2.2 \\
\hline & & & & 3 & $1.87-1.90$ & $-1.80-1.93$ & 2.3 \\
\hline & & & & 4 & $1.87-1.90$ & $-1.80-1.93$ & 2.3 \\
\hline \multirow[t]{3}{*}{ A5 } & $1.8 \times 33$ & 4.8 & 0.084 & 1 & $1.90-1.92$ & $-1.80-1.80$ & 1.7 \\
\hline & & & & 2 & $1.90-1.92$ & $-1.80-1.80$ & 1.7 \\
\hline & & & & 3 & $1.90-1.92$ & -1.80-1.80 & 1.8 \\
\hline
\end{tabular}

a P: Prostate VMAT cases; B: Brain VMAT cases; A: Anal VMAT cases.

b Leaf travel and modulation complexity score (LTMCS) for a VMAT plan. LTMCS ranges from 0 to 1. Low LTMCS indicates high modulation complexity.

Epiqa ${ }^{\mathrm{TM}} \cdot{ }^{11}$ All detectors demonstrated low pass rate failure to the $2 \mathrm{~mm}$ widening of both MLC banks, and this error has the least dosimetric impact on the plans. In addition, several studies have stated poor correlation between the gamma index pass rates of QA procedure and DVH deviations. ${ }^{12,13}$

Although previous studies have studied both systematic and random MLC leaf errors in VMAT plans, in actual beam delivery, if the difference between actual leaf position and planned one is larger than $2 \mathrm{~mm}$, the LINAC will trigger an MLC interlock which invokes a "beam hold-off". ${ }^{14}$ Therefore, the purpose of this study is to evaluate the dosimetric error of clinical VMAT plans caused by MLC leaf position errors that will not trigger
MLC interlock (i.e. beam hold-off). In addition, we evaluated the sensitivity of patient-specific IMRT QA method using MapCHECK ${ }^{\circledR 2}$ for VMAT plans with non-beam-hold MLC errors.

\section{Methods}

\section{A. Patient selection and plan complexity evaluation}

We selected 11 VMAT plans (Table 1) on three types of targets: anus, brain and prostate. Leaf travel and modulation complexity score (LTMCS) was used to characterize the modulation complexity of each VMAT plan. ${ }^{15}$ LTMCS ranges from 0 to 1 and 
it approaches 0 for increasing degree of modulation and increasing total leaf travel distance. In this study, anal VMAT plans had low LTMCS while brain and prostate VMAT plans had moderate to high LTMCS (Table 1).

LTMCS is derived by the product of leaf travel index (LTi) and modulation complexity score (MCSv). ${ }^{15,16}$ MCSv was derived by Masi et al to characterize the modulation degree of the MLC leaves of a VMAT plan. MCSv value of 1 indicates no modulation by MLC leaves (i.e. a plan of the least complexity), and the value decreases as modulation complexity increases. Total travel distance of all in-field moving MLC leaves was calculated and normalized to acquire LTi. When LTi approaches 1 , it indicates short travel distance of all in-field moving MLC leaves. LTi decreases to zero as total leaf travel distance increases.

\section{B. MLC leaf speed modifications}

All original treatment plan DICOM files were exported from the Eclipse ${ }^{\mathrm{TM}}$ (version 10.0, Varian Medical Systems, Inc., Palo Alto, USA) treatment planning system (TPS). The DICOM plans were modified through an in-house developed software. For each arc in the VMAT plan, in-field moving MLC leaves of 178 control points (CPs) on both banks were selected for leaf speed modifications. Speed of each moving MLC leaf per CP was calculated based on MLC leaf position, gantry rotation angle and gantry speed as shown in Equation [1].

$V_{\text {leaf }}(m, n)=\frac{L P(m, n+1)-L P(m, n)}{\Delta t(n)}$

where $\Delta t(n)=\frac{\theta(n+1)-\theta(n)}{u(n)}$

and m: MLC leaf $\epsilon[1,120] ; \quad \mathrm{n}: \mathrm{CP} \in[0,177]$.

Here $\Delta t(n)$ is the gantry rotation time between two adjacent CPs, $\theta(n)$ is the gantry angle of CP ' $n$ ', $u(n)$ is the gantry speed of $C P$ ' $n$ ', $V_{\text {leaf }}(m, n)$ is the speed of $m^{\text {th }}$ leaf of $C P$ ' $n$ ' and $\operatorname{LP}(m, n)$ is the position of $\mathrm{m}^{\text {th }}$ leaf of $C P$ ' $n$ '. MLC leaves on bank 'A' were marked from 1 to 60 while those on bank 'B' were marked from 61 to 120.

For each CP of the arc, leaves on both banks (MLC leaf: 1-120) with the highest speed were set to move at $2.3 \mathrm{~cm} / \mathrm{s}$, resulting in a leaf position difference at a maximum of $2 \mathrm{~mm}$ (Table 1: Range of leaf position chages along $178 \mathrm{CPS}$ ). New leaf position of $C P$ ' $n$ ' is:

$L P_{\text {new }}(m, n)=L P(m, n-1)+2.3(\mathrm{~cm} / \mathrm{s}) \times \Delta t(n-1)$.

If one leaf was moving with the highest speed at two consecutive CPs, leaf motion direction of each $\mathrm{CP}$ was further considered as shown below:

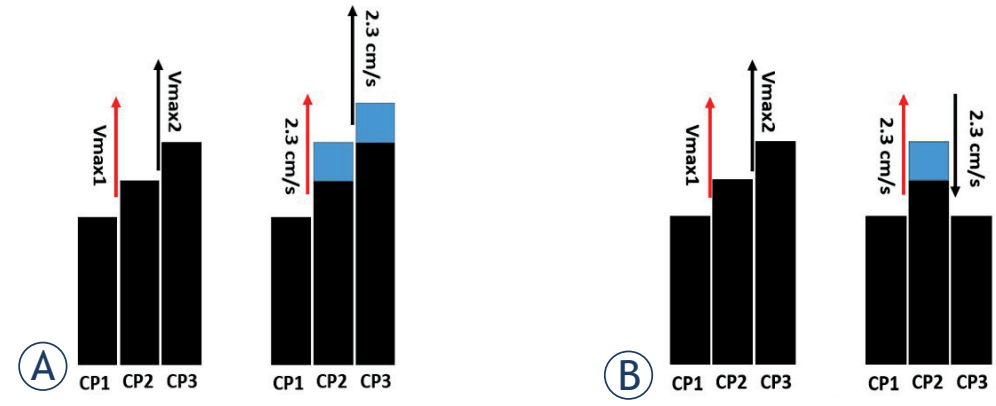

FIGURE 1. Illustration of multi-leaf collimator (MLC) leaf position modifications of one leaf when it is moving with the highest speed at two consecutive control points (CPs) (i.e. CP2 and CP3). (A) The leaf is moving in the same direction. (B) The leaf is moving back and forth. Red arrow ( $V$ maxl or $2.3 \mathrm{~cm} / \mathrm{s}$ ) represents leaf speed from $\mathrm{CP} 1$ to $\mathrm{CP} 2$; Black arrow (Vmax2 or $2.3 \mathrm{~cm} / \mathrm{s}$ ) represents leaf speed from CP2 to CP3. Black bars represent original leaf positions. Blue bars represent new leaf positions after modification.

1. If the motion directions of the next two CPs remained the same, then both leaf positions of corresponding $\mathrm{CP}$ were subject to modification (Figure 1A).

2. If the motion directions of the next two CPs were different, we only modified the leaf position of the middle CP so that both leaf speed values would be increased (Figure 1B).

The total modified MLC leaves percentage (Equation 3 and Table 1) was an indicator of the amount of MLC leaves that had been changed to the maximal speed in each arc;

Total modified MLC leaves $\%=$

$100 \% \times \frac{\sum_{i=1}^{178} \text { Modified in field leaves on both banks of CP } i}{\sum_{i=1}^{178} \text { Total in field moving leaves on both banks of } \mathrm{CP} i}$

where modified in field leaves on both banks of CPi is the total modified MLC leaves that moving with the highest speed of current CP. Leaf speed modification would not be applied if it caused any leaf pair collision (Gap between leaf pair should be no less than $0.5 \mathrm{~mm}$ in actual delivery).

In this study, modified plans were considered as 'standard' plans where MLC leaves were allowed to move at the maximal speed $(2.3 \mathrm{~cm} / \mathrm{s})$. The original plans were considered as 'slowing MLC' plans where the highest MLC speed was lower than 2.3 $\mathrm{cm} / \mathrm{s}$. There were no changes in monitor unit (MU) and gantry speed per CP in all modified VMAT plans.

\section{MLC leaf speed change evaluation}

Having increased the leaf speed of one $\mathrm{CP}$ to the maximal limit without triggering the MLC error interlock (i.e. MLC leaf position difference was less than $2 \mathrm{~mm}$ ), leaf speed of the next CP would be 
TABLE 2. Demonstration of the impact on leaf speed of adjacent control points (CPs) due to leaf modifications

\begin{tabular}{cccccc}
\hline Scenario & $\begin{array}{c}\text { LP1 } \\
(\mathrm{cm})\end{array}$ & $\begin{array}{c}\text { LP2 } \\
(\mathrm{cm})\end{array}$ & $\begin{array}{c}\text { LP3 } \\
(\mathbf{c m})\end{array}$ & $\begin{array}{c}\text { LP1-2 Speed } \\
(\mathrm{cm} / \mathrm{s})\end{array}$ & $\begin{array}{c}\text { LP 2-3 Speed } \\
(\mathrm{cm} / \mathrm{s})\end{array}$ \\
\hline A (ori) & 4.6 & 5.4 & 5.9 & 1.8 & 1.1 \\
A (mod) & 4.6 & 5.6 & 5.9 & $\mathbf{2 . 3}$ & $\mathbf{0 . 7}$ \\
B (ori) & 4.6 & 5.4 & 4.9 & 1.8 & -1.1 \\
B (mod) & 4.6 & 5.6 & 4.9 & $\mathbf{2 . 3}$ & $\mathbf{- 1 . 6}$ \\
\hline
\end{tabular}

a Scenario A: Leaf moved forward from LP1 to LP2, then moved forward from LP2 to LP3. Scenario B: Leaf moved forward from LP1 to LP2, then moved backward from LP2 to LP3.

In the table, 'LPn': leaf position at CP ' $n$ ' =1,2,3; 'ori': original leaf positions; 'mod': leaf positions after modification; positive speed: leaf moved forward; negative speed: leaf moved backward. The speed was the distance between $\mathrm{LPl}, 2,3$ divided by $\Delta t=0.435 \mathrm{~s}$. For both scenarios $\mathrm{A}$ and $\mathrm{B}$, we only modified LP2 from 5.4 to 5.6 to increase LP1-2 speed from $1.8 \mathrm{~cm} / \mathrm{s}$ to $2.3 \mathrm{~cm} / \mathrm{s}$.

affected by this modification. With $\Delta \mathrm{t}(\mathrm{n})$ of each $\mathrm{CP}$ remained unchanged, increasing leaf speed by modifying leaf position of current $\mathrm{CP}$ while keeping the leaf position of the next $\mathrm{CP}$ unchanged resulted in consequential change of leaf speed of the next CP. As a result, total number of leaf speed changes in one arc is twice as many as the total number of MLC leaves that were set to the maximal leaf speed (see Equation [4] below). This accompanied effect caused by the MLC leaf modification either increases or decreases the MLC leaf speed of the next $\mathrm{CP}$ according to the leaf motion direction (Table 2). We have taken this accompanied leaf speed modification into account when evaluating dosimetric changes.

Because of the high complexity (i.e. low LTMCS) of the anal VMAT plans, we further analyzed MLC leaf changes in these VMAT plans. The average percentage of modified MLC leaves (Table 3) was the summation of total percentage of modified MLC leaves for all arcs (Table 1: Total Modified MLC leaves \%) divided by number of total arcs in the plan. The average percentage of faster moving leaves (Faster Moving Leaves \%) depends on MLC leaves that were set to the maximal speed and total faster moving leaves after modifications including those modified leaves of current $\mathrm{CP}$ and affected leaves of the next CP (Equation [4]);

$$
\begin{aligned}
& \overline{\text { Faster Moving Leaves }} \%= \\
& \frac{\sum_{a r c=1}^{n} \text { leaves of higher speed after modifications }}{\sum_{a r c=1}^{n} 2 \times M L C \text { leaves set to the maximal speed }} \times 100 \%
\end{aligned}
$$

where $\mathrm{n}$ is the number of total arcs.

\section{Planar dose measuring system}

In this study, we used MapCHECK ${ }^{\circledR} 2$ 2D diode array system (Model 1177, Sun Nuclear Co.,
Melbourne, FL) for evaluating the effect of slowing MLC leaves on planar dose delivery accuracy. MapCHECK ${ }^{\circledR} 2$ along with its software have been widely used as the clinical implementation for patient-specific verification of VMAT plans due to its compact diode size $(0.8 \mathrm{~mm} \times 0.8 \mathrm{~mm})$, dose linearity, real-time measurement, reproducibility and sensitivity. ${ }^{17-21}$

\section{E. Dosimetric evaluation \\ E.1 Measurement and uncertainty evaluation}

All the VMAT plans ('standard' and 'slowing MLC' plans) were delivered using a Varian Trilogy ${ }^{\circledR}$ LINAC on the same day. Measurement of each arc was then compared with the corresponding calculated planar dose from the TPS with respect to absolute dose Van Dyk distance-to-agreement (DTA) comparison (dose difference is normalized to global maximum) using 3\%/3 mm criteria.. ${ }^{22}$ All measurements were repeated on two consecutive days. The uncertainty was then obtained by evaluating the variation in repeated measurements.

\section{E.2 Dosimetric evaluation of 'slowing MLC' plans}

According to current pre-treatment IMRT QA method for VMAT plans with MapCHECK ${ }^{\circledR}$ 2, measurement of each arc in the 'standard' plan was compared with calculated planar dose of the 'standard' plan with respect to absolute dose Van Dyk DTA comparison using 3\%/3 mm and 2\%/2 $\mathrm{mm}$ criteria. Pass rate $\left(P S_{\text {standard }}\right)$ of the comparison was demonstrated in percentage. $P S_{\text {standard }}$ of each arc using $3 \% / 3 \mathrm{~mm}$ criteria was used as a baseline to verify that all the plan parameters had been correctly transferred from control console computer to LINAC for delivery.

Each 'slowing MLC' plan was considered as a 'standard' plan with MLC leaf errors that would not trigger any MLC interlock to interrupt the beam delivery. In order to evaluate the sensitivity of the IMRT QA method for VMAT plans with nonbeam-hold leaf errors, we delivered each 'slowing MLC' plan and compared the measurement with calcuated planar dose of the 'standard' plan with respect to absolute dose Van Dyk DTA comparison using $3 \% / 3 \mathrm{~mm}$ and $2 \% / 2 \mathrm{~mm}$ criteria to acquire the pass rate $\left(P S_{\text {slowing MLC }}\right)$ in percentage. Because of the MLC leaf errors in each 'slowing MLC' plan, there was a decrease in pass rate of each arc (Equation [5]).

Decrease in pass rate of specific arc $(\%)=$

$\left|P S_{\text {standard }}(\%)-P S_{\text {slowng } M L C}(\%)\right|$ 


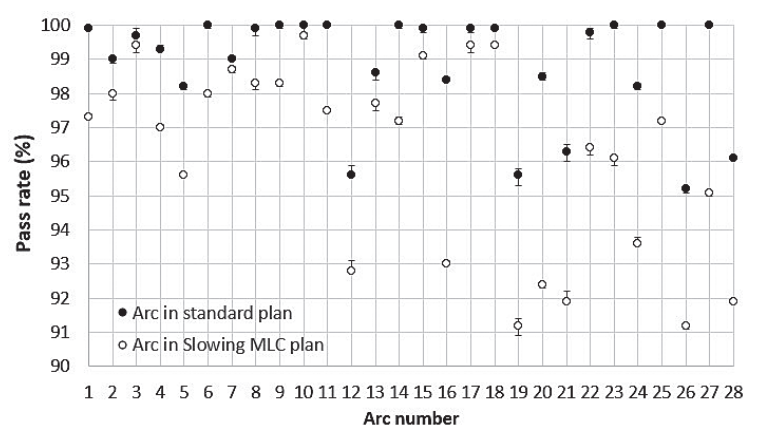

FIGURE 2. Variation of pass rates $(3 \% / 3 \mathrm{~mm})$ of each volumetric modulated arc therapy (VMAT) arc. Solid dots: pass rates of arcs in 'standard' plans ( $\left.P S_{\text {standara }}\right)$. Soft dots: pass rates of arcs in 'slowing multi-leaf collimator (MLC)' plans (PS slowing MLC). Error bars are pass rates variation based on repeated measurements of each arc on two consecutive days.

The correlations between the decreases in pass rates using $3 \% / 3 \mathrm{~mm}$ and $2 \% / 2 \mathrm{~mm}$ criteria and LTMCS were analyzed through Spearman's correlation coefficient. ${ }^{23}$

Finally, the 3D dose distribution of each plan was calculated in the TPS and dose-volume histogram (DVH) for targets and organs-at-risk (OAR) were obtained. For clinical dosimetric evaluation, mean target dose (Dmean), dose that covers 95\% $\left(D_{95}\right)$ of the planning target volume, and Normal Tissue Complication Probability (NTCP) using Lyman Kutcher Burman (LKB) model ${ }^{23}$ were calculated for all the plans. Clinical dosimetric parameters of 'standard' and 'slowing MLC' plans were compared using the Wilcoxon signed-rank test. ${ }^{24}$

\section{Results}

\section{A. Pass rate and uncertainty evaluation}

Figure 2 demonstrated pass rate of each arc and variation of measurements based on repeated measurements on two consecutive days. Among all the arcs in both 'standard' and 'slowing MLC' plans, the maximal variation found was $0.3 \%$ with respect to the $91.5 \%$ pass rate.

\section{B. Prostate cases}

For all three prostate cases, $P S_{\text {standard }}$ and $P S_{\text {slowing }}$ MLC using $3 \% / 3 \mathrm{~mm}$ and $2 \% / 2 \mathrm{~mm}$ criteria were all higher than 90\% (Figure 3 and 4: Prostate). Dosimetric differences of Dmean, D95, NTCP (bladder, rectum, leaf and right femoral heads) between 'slowing MLC' plans and 'standard' plans were: $0.47 \pm 0.17$ Gy $(p>0.05), 0.33 \pm 0.13$ Gy ( $p$
TABLE 3. Target dose differences between 'standard' and 'slowing multi-leaf collimator (MLC)' anal volumetric modulated arc therapy (VMAT) plans, total leave states, and average percentages of modified leaves and faster moving leaves of anal cases

\begin{tabular}{|c|c|c|c|c|c|}
\hline Case & $\triangle D$ mean $(G y)^{a}$ & $\triangle \mathrm{D} 95(\mathrm{~Gy})^{\mathrm{a}}$ & $\begin{array}{c}\text { Total leave states } \\
\text { per arc (leaves } \\
\text { on both banks } \\
/ C P) \times(C P)\end{array}$ & $\begin{array}{c}\text { Average } \\
\text { modified } \\
\text { MLC leaves } \\
(\%) \\
\end{array}$ & $\begin{array}{c}\text { Average } \\
\text { faster } \\
\text { moving } \\
\text { leaves (\%) }\end{array}$ \\
\hline $\mathrm{Al}$ & -0.8 & -0.2 & $120 \times 178$ & 1.7 & 56.5 \\
\hline $\mathrm{A} 2$ & -0.9 & -0.3 & $120 \times 178$ & 1.6 & 53.6 \\
\hline A3 & -1.2 & -0.5 & $120 \times 178$ & 1.7 & 51.3 \\
\hline A4 & -2.2 & -1.0 & $120 \times 178$ & 2.3 & 69.0 \\
\hline A5 & -1.1 & -0.3 & $120 \times 178$ & 1.7 & 52.7 \\
\hline
\end{tabular}

a Negative sign means dose of 'standard' plan is lower than that of 'slowing MLC' plan $\mathrm{CP}=$ control point

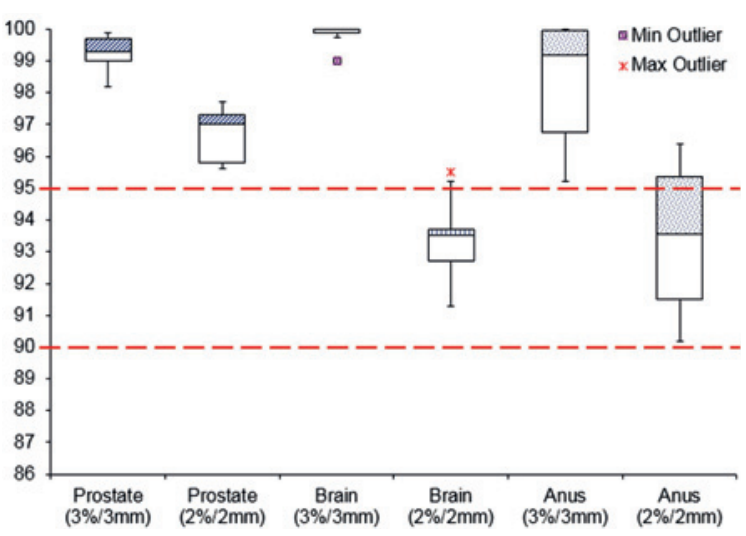

FIGURE 3. Pass rates of 'standard' volumetric modulated arc therapy (VMAT) plans with respect to absolute dose Van Dyk distance-to-agreement (DTA) comparisons using $3 \% / 3 \mathrm{~mm}$ and $2 \% / 2 \mathrm{~mm}$ criteria.

$>0.05), 1 \% \pm 1 \%_{\text {bladder }}(\mathrm{p}>0.05), 3 \% \pm 2 \%_{\text {rectum }}(\mathrm{p}>$ $0.05), 2 \% \pm 1 \%_{\text {left fem }}(\mathrm{p}>0.05), 2 \% \pm 1 \%_{\text {right fem }}(\mathrm{p}>$ $0.05)$, respectively.

\section{Brain cases}

For all three brain cases, $P S_{\text {standard }}$ and $P S_{\text {slowing MLC }}$ using $3 \% / 3 \mathrm{~mm}$ and $2 \% / 2 \mathrm{~mm}$ criteria were all higher than 90\% (Figure 3 and 4: Brain) except for arc 2 of brain case B2. Dosimetric differences of Dmean, D95, NTCP (brain stem, cerebellum, spinal cord, left and right cochlea) between 'slowing MLC' plans and 'standard' plans were: $0.13 \pm 0.05$ Gy ( $p>0.05), 0.17 \pm 0.09$ Gy ( $p>0.05), 1 \% \pm 1 \%_{\text {brain }}$ stem $(p>0.05), 1 \% \pm 1 \%_{\text {cerebellum }}(p>0.05), 0 \% \pm 1 \%_{\text {spinal }}$ cord $(p>0.05), 1 \% \pm 2 \%_{\text {left cochlea }}(p>0.05), 1 \% \pm 1 \%_{\text {right }}$ cochlea $(p>0.05)$, respectively. 


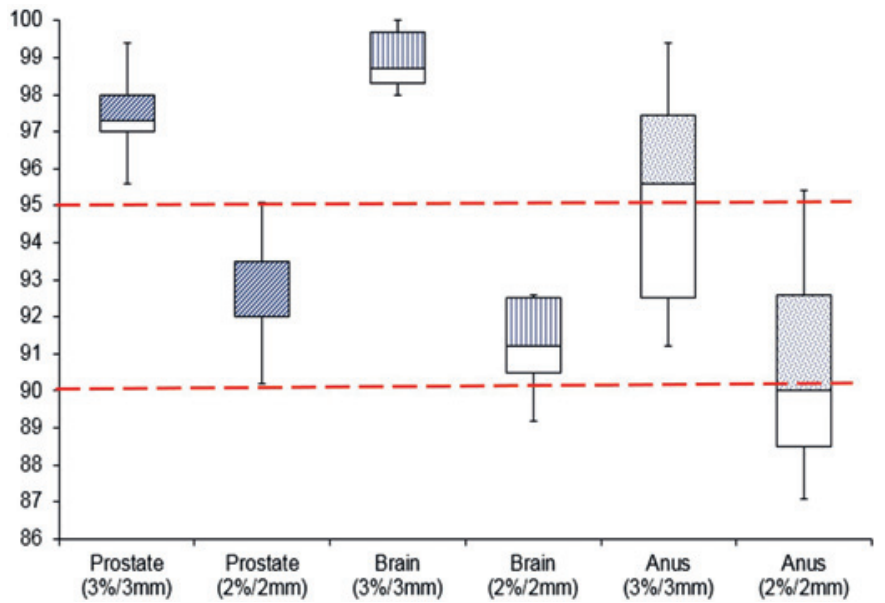

FIGURE 4. Pass rates of 'slowing multi-leaf collimator (MLC)' volumetric modulated arc therapy (VMAT) plans with respect to absolute dose Van Dyk distance-to-agreement (DTA) comparisons using $3 \% / 3 \mathrm{~mm}$ and $2 \% / 2$ $\mathrm{mm}$ criteria.
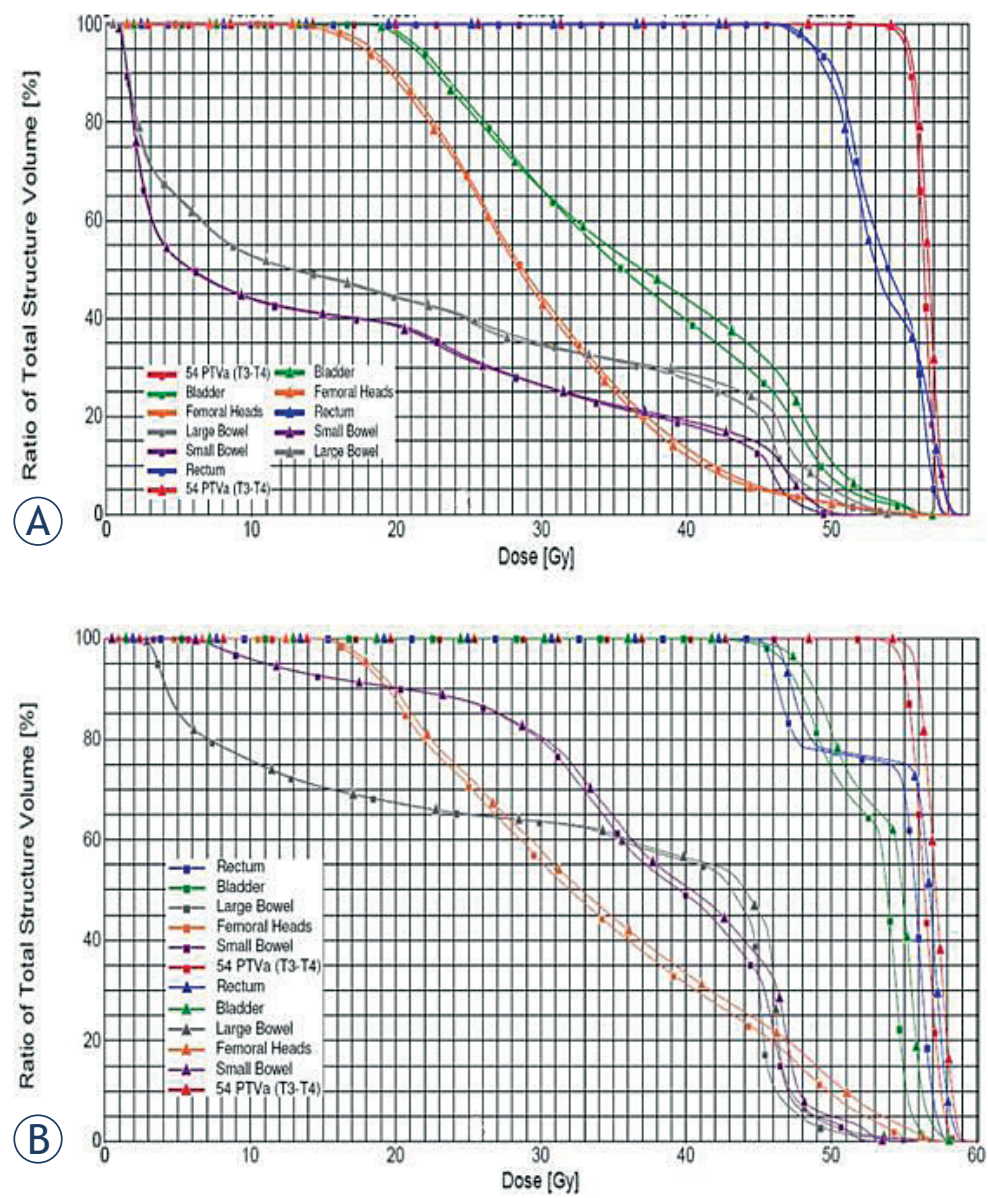

FIGURE 5 (A) DVH comparison between 'standard' and 'slowing MLC' VMAT plans of anal case A3. (B) DVH comparison between 'standard' and 'slowing MLC' VMAT plans of anal case A4. $\mathbf{\Delta}$ :'slowing MLC' plan; - :standard' plan. Red: PTV; Blue: Rectum; Green: Bladder; Grey: Large bowel; Purple: Small bowel; Orange: Femoral heads

\section{Anal cases}

The LTMCS scores of Anal VMAT plans were smaller than both brain and prostate VMAT plans (Table 1: LTMCS) indicating higher modulation by MLC leaves. For anal VMAT cases, $P S_{\text {standard }}$ and $P S_{\text {slowing MLC }}$ using $3 \% / 3 \mathrm{~mm}$ criteria were all

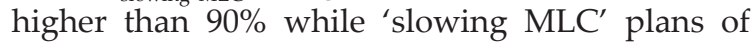
cases A3, A4 and A5 demonstrated less than 90\% pass rates using $2 \% / 2 \mathrm{~mm}$ criteria (Figure 4: Anus). Dosimetric differences of NTCP (bladder, rectum, large bowel and femoral heads) between 'slowing MLC' plans and 'standard' plans were: $2 \% \pm 2 \%{ }_{\text {blad- }}$ der $(p>0.05), 3 \% \pm 1 \%_{\text {rectum }}(p>0.05), 2 \% \pm 1 \%_{\text {large }}$ bowel $(p>0.05), 1 \% \pm 1 \%_{\text {femheads }}(p>0.05)$, respectively. Compared with anal case A3 and A5, case A4 demonstrated substantial dosimetric differences between the 'standard' and 'slowing MLC' plans where $\Delta D_{\text {mean }}$ and $\Delta D_{95}$ were 2.2 Gy and 1.0 Gy respectively (Figure 5 and Table 3 ).

\section{E. Correlation between LTMCS and dosimetric parameters}

The correlation between decreases in pass rates of VMAT arcs using $2 \% / 2 \mathrm{~mm}$ criteria and LTMCS is moderate to strong $\left(r_{s}=0.597\right.$, Figure $\left.6 \mathrm{~A}\right)$. When using $3 \% / 3 \mathrm{~mm}$ criteria, the correlation is weak to moderate $\left(r_{s}=0.453\right.$, Figure $\left.6 B\right)$.

\section{Discussion}

\section{A. Measurement uncertainty}

By using lasers and front pointer for device positioning, the measurement setup was of high consistency. Absolute dose calibration for MapCHECK ${ }^{\circledR 2}$ was performed every day before dose measurement. ${ }^{25,26}$ Therefore, the source of the uncertainty is mainly due to variability of MLC leaf motion. The small error bars in Figure 2 indicate that the measurement variability is very small.

\section{B. Anal case A4 results}

For anal case A4, since the MU of each control point remained unchanged, and 'slowing MLC' plan had more slowly moving MLC leaves, more area were being irradiated that resulted in higher dose. The average percentage of faster moving leaves indicates the amount of MLC leaves moving back-and-forth. The average percentages of modified MLC leaves (2.3\%) and average percentage of faster moving leaves (69\%) of anal case A4 are higher compared with other anal 

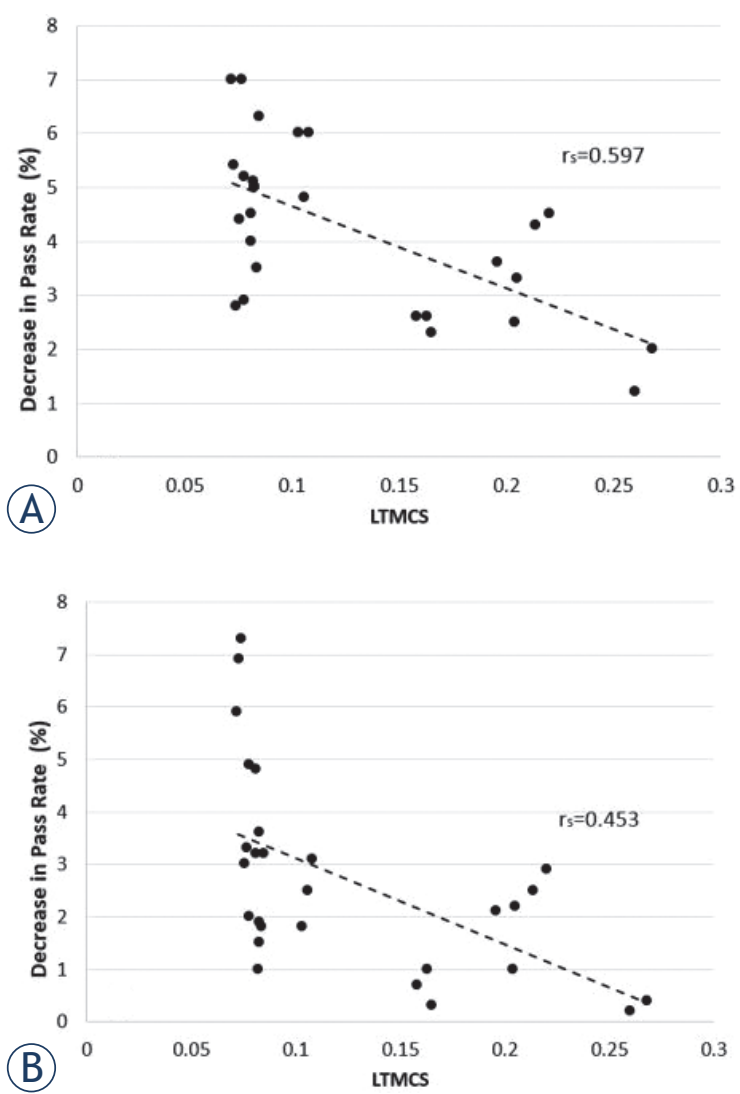

FIGURE 6 Correlation between absolute pass rate difference $\left(\left|P S_{\text {standard }}(\%)-P S_{\text {slowng MLC }}(\%)\right|\right)$ of each arc and LTMCS of each arc. (A) LTMCS vs decrease in pass rate (\%) using $2 \% / 2 \mathrm{~mm}$ criteria; (B) LTMCS vs decrease in pass rate (\%) using $3 \% / 3 \mathrm{~mm}$ criteria

cases (Table 3), indicating that more high speed MLC leaves were moving back and forth to create a highly modulated VMAT plan. Accordingly, the anal case A4 has the minimal LTMCS among all anal cases studied.

Moreover, all four arcs of anal case A4 have large fields (e.g. $14 \mathrm{~cm} \times 30 \mathrm{~cm}, 14 \mathrm{~cm} \times 29 \mathrm{~cm}, 30 \mathrm{~cm}$ $\times 14 \mathrm{~cm}, 30 \mathrm{~cm} \times 14 \mathrm{~cm}$ for arc 1, 2, 3, 4 respectively). Wijesooriya et al reported the accuracy of RapidArc delivery holds for leaf velocities with small dosimetric uncertainties for $5 \mathrm{~mm}$ width MLC leaves which are in the central $20 \mathrm{~cm}$ of field. ${ }^{7}$ They found that three VMAT plans with large MLC leaves with $1 \mathrm{~cm}$ width at high speed $(2.1-2.4 \mathrm{~cm} / \mathrm{s})$ demonstrated higher leaf position inaccuracy. Therefore, large MLC leaves in the VMAT plan of anal case A4 have more effect on dose delivery inaccuracy.

\section{Pass rates and dosimetric parameters}

When using 3\%/3 mm criteria, all 11 cases including 'standard' and 'slowing MLC' plans passed the

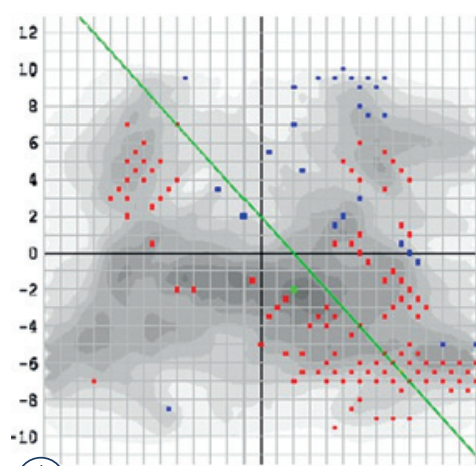

(A)

FIGURE 7 MapCHECK ${ }^{\circledR} 2$ measurements of single arc of case A4. (A) Red dots are MapCHECK ${ }^{2} 2$ measurements of 'slowing MLC' plan showing delivered dose is higher than planed dose ('standard' plan); (B) Dotted line represents dose profile of 'slowing MLC' plan; Black solid line represents dose profile of 'standard' plan.

institutional $90 \%$ acceptance threshold of absolute dose DTA comparison. Dosimetric differences (e.g. $\Delta D_{\text {mean }}, \Delta D_{95}$ and NTCP) between 'standard' and 'slowing MLC' plans in targets and normal tissues were minimal indicating that VMAT plans with non-beam-hold MLC leaf errors (leaf position difference $\leq 2 \mathrm{~mm}$ ) remain the planned dose coverage except for anal case A4. Using $2 \% / 2 \mathrm{~mm}$ criteria, decrease in pass rates of VMAT arcs demonstrated stronger correlation with VMAT modulation complexity which is characterized by LTMCS (Figure 6A).

Some arcs in 'slowing MLC' plans of anal cases A3 and A5 showed less than $90 \%$ pass rates using $2 \% / 2 \mathrm{~mm}$ criteria although differences in dosimetric parameters are small (e.g. $\Delta D_{\text {mean }}$ and $\Delta D_{95}$. However, anal case A4 showed a consistent decrease in pass rates and dose conformity. Compared with the 'standard' plan, 'slowing MLC' plan of anal case A4 delivered higher planar dose (Figure 7) which is consistent with the changes in DVH curves in Figure 5B. To further ensure the dosimetric quality of VMAT plans like anal case A4 that have the following features: 1 . Highly modulated multiple arc VMAT plan (e.g. LTMCS $<0.1$ ); and 2. Arc has large field size that involves more thick MLC leaves, we recommend $2 \% / 2 \mathrm{~mm}$ criteria for absolute dose Van Dyk DTA comparison which is more sensitive to non-beam-hold leaf position errors.

\section{Conclusions}

For ten out of eleven cases, DVH comparisons between 'standard' and 'slowing MLC' VMAT plans 
demonstrated minimal dosimetric changes in targets and OAR. Pass rate threshold (90\%) using $3 \% / 3 \mathrm{~mm}$ criteria is not sensitive in detecting MLC leaf errors that will not trigger the MLC leaf interlock. However, the consequential effects on target and OAR are negligible, which supports the reliability of current IMRT QA method for VMAT plan verification.

\section{References}

1. Clivio A, Fogliata A, Franzett PA, Nicolini G, Vanetti E, Wyttenbach R, et al Volumetric-modulated arc radiotherapy for carcinomas of the anal canal: A treatment planning comparison with fixed field IMRT. Radiother Oncol 2009; 92: 118-24.

2. Sale C, Moloney P. Dose comparisons for conformal, IMRT and VMAT prostate plans. J Med Imaging Radiat Oncol 2011; 55: 611-21.

3. Ling CC, Zhang P, Archambault Y, Bocanek J, Tang G, Losasso T. Commissioning and quality assurance of RapidArc radiotherapy delivery system. Int J Radiat Oncol Biol Phys 2008; 72: 575-81.

4. Tatsumi D, Hosono MN, Nakada N, Ishii K, Tsutsumi S, Inoue M, et al. Direct impact analysis of multi-leaf collimator leaf position errors on dose distributions in volumetric modulated arc therapy: a pass rate calculation between measured planar doses with and without the position errors. Phys Med Biol 2011; 56: N237-46.

5. Peng J, Zhang Z, Zhou L, Zhao J, Wang J, Kong L, et al. A study on investigating the delivery parameter error effect on the variation of patient quality assurance during RapidArc treatment. Med Phys 2013; 40: 031703.

6. Oliver M, Gagne I, Bush K, Zavgorodni S, Ansbacher W, Beckham W. Clinica significance of multi-leaf collimator positional errors for volumetric modulated arc therapy. Radiother Oncol 2010; 97: 554-60.

7. Wijesooriya K, Aliotta E, Benedict S, Read P, Rich T, Larner J. RapidArc patient specific mechanical delivery accuracy under extreme mechanical limits using LINAC log files. Med Phys 2012; 39: 1846-53.

8. Arumugam S, Xing A, Goozee G, Holloway L. Detecting VMAT delivery errors: a study on the sensitivity of the ArCCHECK-3D electronic dosimeter. Journal of Physics: Conference Serire 444(1) 2013; 1-4.

9. Schreibmann E, Dhabaan A, Elder E, Fox T. Patient-specific quality assurance method for VMAT treatment delivery. Med Phys 2009; 36: 4530.

10. Bakhtiari M, Kumaraswamy L, Bailey DW, deBoer S, Malhotra HK, Podgorsak MB. Using an EPID for patient-specific VMAT quality assurance. Med Phys 2011; 38: 1366-73.

11. Fredh A, Scherman JB, Fog LS, Munck af RP. Patient QA systems for rotational radiation therapy: a comparative experimental study with intentional errors. Med Phys 2013; 40: 031716.

12. Zhen $\mathrm{H}$, Nelms $\mathrm{BE}$, Tome WA. Moving from gamma pass rates to patient DVH-based QA metrics in pretreatment dose QA. Med Phys 2011; 38: 5477-89.

13. Oliver M, Bush K, Zavgorodni S, Ansbacher W, Beckham WA. Understanding the impact of RapidArc therapy delivery errors for prostate cancer. J App / Clin Med Phys 2011; 12: 32-43.

14. LoSasso T, Chui CS, Ling CC. Comprehensive quality assurance for the delivery of intensity modulated radiotherapy with a multileaf collimator used in the dynamic mode. Med Phys 2001; 11: 2209-19.

15. McNiven AL, Sharpe MB, Purdie TG. A new metric for assessing IMRT modulation complexity and plan deliverability. Med Phys 2010; 37: 505

16. Masi L, Doro R, Favuzzaet V, Cipressi S, Livi L. Impact of plan parameters on the dosimetric accuracy of volumetric modulated arc therapy. Med Phys 2013; 40: 071718.

17. Iftimia I, Cirino ET, Xiong L, Mower HW. Quality assurance for methodology for Varian RapidArc treatment plans. J Appl Clin Med Phys 2010; 11: 130-43.
18. Gloi AM, Buchanan RE, Zuge CL, Goettler AM. RapidArc quality assurance through MapCHECK. J Appl Clin Med Phys 2011; 12: 39-47.

19. Jursinic PA, Sharma R, Reuter J. MapCHECK used for rotational IMRT measurements: step-and-shoot, TomoTherapy, RapidArc. Med Phys 2010; 37: 2837-46.

20. Rinaldin G, Perna L, Agnello G, Pallazzi G, Cattaneo GM, Fiorino C, et al. Quality assurance of RapidArc treatments: performances and pre-clinical verifications of a planar detector (MapCHECK2). Phys Medica 2014; 30:18490.

21. Shim SJ, Shim JB, Lee SH. Quality assurance of volumetric modulated arc therapy for Elekta Synergy. Korean J Med Phys 2012; 23: 33-41.

22. Van Dyk J, Barnett RB, Cygler JE, Shragge PC. Commissioning and quality assurance of treatment planning computers. Int J Radiat Oncol Biol Phys 1993; 26: 261-73.

23. Lyman JT. Complication probability as assessed from dose-volume histograms. Radiat Res 1985; 104: 13-9.

24. Chaikh A, Giraud JY, Perrin E, Bresciani JP, Balosso J. The choice of statistical methods for comparisons of dosimetric data in radiotherapy. Radiat Oncol 2014; 9: 205.

25. Jursinic PA, Nelms BE. A 2D diode array and analysis software for verification of intensity modulated radiation therapy delivery. Med Phys 2003; 30: $870-9$

26. Letourneau D, Gulam M, Yan D, Oldham M, Wong JW. Evaluation of a 2D diode array for IMRT quality assurance. Radioth Oncol 2004; 70: 199-206. 